\title{
Validation of EN ISO method 10273-Detection of pathogenic Yersinia enterocolitica in foods
}

\section{Interlab Study Grp}

2019-01-02

Interlaboratory study group , Hallanvuo , S , Herranen , M, Jaakkonen , A, Nummela , M , Ranta , J , Botteldoornl , N, De Zutter , L, Fredriksson-Ahomaa , M , Hertwig , S , Johannessen, G S , Ludewig, M, Messelhaeusser , U , Sigvart-Mattila , P ,

Thisted-Lambertz , S , Thure, T \& Vatunen , E 2019, ' Validation of EN ISO method 10273-Detection of pathogenic Yersinia enterocolitica in foods ' , International Journal of Food Microbiology , vol. 288 , pp. 66-74 . https://doi.org/10.1016/j.ijfoodmicro.2018.01.009

http://hdl.handle.net/10138/324233

https://doi.org/10.1016/j.ijfoodmicro.2018.01.009

cc_by_nc_nd

acceptedVersion

Downloaded from Helda, University of Helsinki institutional repository.

This is an electronic reprint of the original article.

This reprint may differ from the original in pagination and typographic detail.

Please cite the original version. 
Elsevier Editorial System(tm) for

International Journal of Food Microbiology

Manuscript Draft

Manuscript Number:

Title: Validation of ISO method 10273 - Detection of pathogenic Yersinia enterocolitica in foods

Article Type: SI: Reference methods validation

Keywords: validation; pathogenic Y. enterocolitica; detection;

standardization; interlaboratory study.

Corresponding Author: Dr. Saija Hallanvuo, Ph.D.

Corresponding Author's Institution: Finnish Food Safety Authority Evira

First Author: Saija Hallanvuo, Ph.D.

Order of Authors: Saija Hallanvuo, Ph.D.; Mirkka Herranen; Anniina Jaakkonen; Maria Nummela; Jukka Ranta; Interlaboratory study group Interlaboratory study group

Abstract: EN ISO 10273 method for the detection of pathogenic Yersinia enterocolitica in foods was validated in the project Mandate M/381 funded by European Commission. A total of 14 laboratories from five European countries participated in the interlaboratory study (ILS) organized during 2013 and 2014. Before the ILS, the method was revised by an international group of experts and the performance of the revised method was assessed in an ILS study. The results are published as a part of the standard EN ISO 10273 revision. The study included three rounds with different sample types; raw milk, iceberg lettuce and minced meat, inoculated with a low and high level of pathogenic $y$. enterocolitica strains representing major pathogenic bioserotypes 4/0:3 and 2/0:9. The homogeneity and stability of the samples were verified before dispatching them to the laboratories. The results demonstrated the method sensitivity of $96 \%$ in raw milk, $97 \%$ in minced meat, and 98\% in lettuce at high inoculation level of pathogenic $Y$. enterocolitica. The specificity was $100 \%$ in raw milk, $96 \%$ in minced meat, and 98\% in lettuce. The level of detection, LOD50, varied between study rounds, being $9.4 \mathrm{CFU} / 25 \mathrm{ml}$ in raw milk, $9.9 \mathrm{CFU} / 25 \mathrm{~g}$ in minced meat and $63 \mathrm{CFU} / 25 \mathrm{~g}$ in lettuce samples. During the study, confirmation by using real-time PCR method ISO/TS 18867 together with pyrazinamidase testing was also validated, as alternative to conventional biochemical confirmation. When comparing different isolation steps used in the revised method during the study rounds, PSB enrichment and plating on CIN after alkaline (KOH) treatment showed the highest sensitivity (52-92\%) in raw milk and minced meat samples. In lettuce samples, however, ITC with $\mathrm{KOH}$ treatment before plating on CIN showed higher sensitivity (64\% at low level; 82\% at high level) than plating on CIN from PSB with KOH treatment (44\% at low level; 74\% at high level). Statistical analysis of different isolation steps supported the use of two enrichment media, PSB and ITC, in the revised method. Recovery of pathogenic $Y$. enterocolitica on CIN was most efficient after $\mathrm{KOH}$ treatment and, based on the analysis, plating on CIN agar without $\mathrm{KOH}$ treatment could be left as optional procedure in the method. 
1 Validation of ISO method 10273 - Detection of pathogenic Yersinia enterocolitica in foods

Saija Hallanvuo ${ }^{a}$, , Mirkka Herranen ${ }^{a 1}$, Anniina Jaakkonen ${ }^{a}$, Maria Nummela ${ }^{a}$, Jukka Ranta ${ }^{b}$ and Interlaboratory study group ${ }^{c}$

Keywords: validation, pathogenic Y. enterocolitica, detection, standardization, interlaboratory study

\section{Abstract}

EN ISO 10273 method for the detection of pathogenic Yersinia enterocolitica in foods was validated in the project Mandate M/381 funded by European Commission. A total of 14 laboratories from five European countries participated in the interlaboratory study (ILS) organized during 2013 and 2014. Before the ILS, the method was revised by an international group of experts and the performance of the revised method was assessed in an ILS study. The results are published as a part of the standard EN ISO 10273 revision. The study included three rounds with different sample types; raw milk, iceberg lettuce and minced meat, inoculated with a low and high level of pathogenic $Y$. enterocolitica strains representing major pathogenic bioserotypes 4/0:3 and 2/0:9. The homogeneity and stability of the samples were verified before dispatching them to the laboratories. The results demonstrated the method sensitivity of $96 \%$ in raw milk, $97 \%$ in minced meat, and $98 \%$ in lettuce at high inoculation level of pathogenic $Y$. enterocolitica. The specificity was $100 \%$ in raw milk, $96 \%$ in minced meat, and $98 \%$ in lettuce. The level of detection, LOD $_{50}$, varied between study rounds, being $9.4 \mathrm{CFU} / 25 \mathrm{ml}$ in raw milk, $9.9 \mathrm{CFU} / 25 \mathrm{~g}$ in minced meat and $63 \mathrm{CFU} / 25$ g in lettuce samples. During the study, confirmation by using real-time PCR method ISO/TS 18867 together with pyrazinamidase testing was also validated, as alternative to conventional biochemical confirmation. When comparing different isolation steps used in the revised method during the study rounds, PSB enrichment and plating on CIN after alkaline $(\mathrm{KOH})$ treatment showed the highest sensitivity (52-92\%) in raw milk and minced meat samples. In lettuce samples, however, ITC with $\mathrm{KOH}$ treatment before plating on CIN showed higher sensitivity (64\% at low level; 82\% at high level) than plating on CIN from PSB with $\mathrm{KOH}$ treatment (44\% at low level; $74 \%$ at high level). Statistical analysis of different isolation steps supported the use of two enrichment media, PSB and ITC, in the revised method. Recovery of pathogenic Y. enterocolitica on $\mathrm{CIN}$ was most efficient after $\mathrm{KOH}$ treatment and, based on the analysis, plating on $\mathrm{CIN}$ agar without $\mathrm{KOH}$ treatment could be left as optional procedure in the method.

\section{Introduction}

The project to elaborate and validate a series of 15 European Standards (EN) in the field of food hygiene legislation was initiated following the agreement signed between the European commission and the European Committee for Standardization (CEN) in December 2010, in response to Mandate M/381. At that time, the standard EN ISO 10273 for the detection of presumptive pathogenic Yersinia enterocolitica in 
foods was last updated in 2003 and lacked collaboratively tested performance criteria. The enrichment and plating steps within the method had been selected on the basis of historical user experience, but not subjected to thorough international collaborative testing.

For the revision and validation of ISO 10273, as part of the Mandate M/381 project, a team of $Y$. enterocolitica methodology experts evaluated and revised the method based on the most recent literature and knowledge of the $Y$. enterocolitica detection methodology. Before revision, the isolation method included enrichment of a test portion in peptone, sorbitol and bile salts (PSB) broth as a tenfold dilution, followed by plating onto Cefsulodin, IrgasanTM and Novobiocin (CIN) agar after potassium hydroxide (KOH) treatment and without $\mathrm{KOH}$ treatment. In parallel, the method included enrichment of a test portion in Irgasan $^{\text {TM }}$, ticarcillin and potassium chlorate (ITC) broth as a 100 -fold dilution, followed by plating onto Salmonella-Shigella-desoxycholate-calcium chloride (SSDC) agar without $\mathrm{KOH}$ treatment (Anonymous 2003).

During the revision, the method was complemented with direct plating on CIN agar, and incubation times were shortened for PSB broth (from 5-6 days to $44 \pm 4 \mathrm{~h}$ ) and CIN agar (from $48 \mathrm{~h}$ to $24 \pm 2 \mathrm{~h}$ ), both of which had been recommended previously (EFSA 2009; Van Damme et al., 2010). In the revised method, 90 $\mathrm{ml}$ of ITC was inoculated with $10 \mathrm{ml}$ of initial suspension in PSB, and SSDC agar was replaced with CIN agar and an optional chromogenic medium. Previous studies on slaughter pig tonsils samples revealed no significant difference between Y. enterocolitica counts on CIN and SSDC media (Van Damme et al., 2010). The performance of chromogenic $Y$. enterocolitica media for qualitative and quantitative isolation of pathogenic $Y$. enterocolitica, however, was equal to SSDC and CIN (Van Damme et al. 2012). Furthermore, direct plating on $\mathrm{CIN}$, or $\mathrm{KOH}$ treatment after ITC enrichment, was recommended to replace the conventional ITC-SSDC step (Van Damme et al., 2012), and thus, $\mathrm{KOH}$ treatment after ITC enrichment was included in the revised method.

For the confirmation of pathogenic $Y$. enterocolitica, tests related to pathogenicity were specified and added in the frontline in the revised method. Determination of pathogenic Yersinia (genus level) involves four tests, while confirmation of pathogenic $Y$. enterocolitica (species level) involves seven tests (conventional biochemical testing) in the revised method. Therefore, it is more convenient to first identify pathogenic Yersinia isolates for further confirmation. Additionally, as the tests related to pathogenicity were made mandatory and allowed the separation of pathogenic from non-pathogenic $Y$. enterocolitica, the word "presumptive" could be replaced with the word "pathogenic" in the standard title.

After the revision, the method was subjected to an interlaboratory study from May 2013 until April 2014, and the results were summarised in EN ISO 10273:2017 (Anonymous, 2017). The aim of the study was to determine sensitivity, specificity, and the level of detection $\left(\operatorname{LOD}_{50}\right)$ of the method. Based on the confirmed 
results of all isolation steps of the method, the necessity of parallel enrichment steps and alkaline treatment in the method were also evaluated. In confirmation of pathogenic $Y$. enterocolitica, the performance of real-time PCR ISO/TS 18867 (Anonymous 2015), together with pyrazinamidase testing, was assessed as an alternative to conventional biochemical confirmation. Furthermore, the performance testing scheme for the quality assurance of the culture media used in the method was verified. We report here the results of the interlaboratory study to validate EN ISO 10273.

\section{Materials and methods}

\subsection{Design of the trial}

A total of 14 laboratories from five European countries (Belgium, Finland, Germany, Norway and Sweden) participated in the interlaboratory study (ILS) organized in 2013 and 2014. The laboratories were experienced in the analysis of pathogenic $Y$. enterocolitica, 11 of the laboratories were accredited according to EN ISO 17025 and three laboratories were established in scientific research of pathogenic $Y$. enterocolitica. The design of the study followed the document ISO/TC34/SC9 N 851 (CEN/TC275/WG6 N 323) "Basis of the mandate study' and included three rounds with different sample matrices; raw milk, minced meat, and green crispy lettuce. Before the ILS, method comparison studies were performed by the organizing laboratory (Finnish Food Safety Authority Evira) during 2011-2013. Furthermore, a pre-trial was organized with 14 participants before the ILS to allow the laboratories to become acquainted with the laboratory procedure and to verify the suitability of the test material for the purpose.

Samples were artificially inoculated to ensure pre-defined inoculation levels and the required homogeneity of samples. For each food type, two inoculum levels; low and high, and blank samples were used based on the preliminary evaluation of the level of detection $\left(\operatorname{LOD}_{50}\right)$ (for inoculum CFU) for each matrix by the organizing laboratory. During each trial round, each participant laboratory analysed 24 randomly coded samples, i.e. 8 replicates at each inoculation level. To ensure that the samples were handled similarly, the laboratories were instructed to start the analyses the day after dispatch, at the earliest (between approximately 18 and $24 \mathrm{~h}$ after dispatch). The laboratories were allowed to start analysing all 24 samples at the same time or in two sets on instructed starting days. Both sets contained an equal number of samples from each level (blank, low and high). The samples were shipped in insulated EPS packages (Biotherm 45 DI 96, DGP Intelsius Ltd. UK) in dry ice and qualified to maintain integrity for $96 \mathrm{~h}$. A temperature data logger (LOG-IC USB Multi Use Logger with Ribbon Probe, American Thermal Instruments, Ohio) was inserted in each package for monitoring of the sample temperature during shipping. In addition, laboratories were asked to record the temperature near the sample tubes when unpacking the samples. The laboratories performed the analyses according to detailed instructions (laboratory procedure) provided 
by the organizer and reported the results on a separate form, including information on factors which may have affected their results (exact incubation times, culture media batches and preparation used, etc.). The protocol used during the ILS study served as the basis for the standard, to which no major technical changes were made before publication.

\subsection{Method used in the interlaboratory study}

The ILS study protocol consisted of the following successive stages for the detection of pathogenic $Y$. enterocolitica:

a) Pre-treatment of food samples according to standardized procedure to obtain initial suspension and inoculation of the suspension (direct plating) onto 2 to 4 selective agar plates (CIN).

b) Enrichment in liquid enrichment medium (PSB) and selective liquid enrichment medium (ITC) at 25 ${ }^{\circ} \mathrm{C}$ for $44 \mathrm{~h}$.

c) Plating out the enrichment with $\mathrm{KOH}$ treatment (mixing $0.5 \mathrm{ml}$ of enrichment in $4.5 \mathrm{ml}$ of $0.5 \% \mathrm{KOH}$ solution for $20 \mathrm{~s}$ ), and without $\mathrm{KOH}$ treatment on CIN agar, followed by incubation at $30^{\circ} \mathrm{C}$ for $24 \mathrm{~h}$ and verification of the colony morphology as presumptive pathogenic $Y$. enterocolitica by successive culturing on selective plates.

d) Confirmation of the presence of pathogenic $Y$. enterocolitica by appropriate biochemical or molecular confirmation tests. The determination of pathogenic Yersinia species was performed first to ease the workload in the following confirmation of pathogenic $Y$. enterocolitica.

In the interlaboratory study, confirmation by using alternative pathway (see Figure S1 in supplementary material) was validated against the conventional biochemical confirmation pathway of ISO 10273. As the alternative pathway, collaborators used real-time PCR for detecting pathogenic $Y$. enterocolitica as described in ISO/TS 18867 (Anonymous 2015), Annex B, Methods 1 or 2 and pyrazinamidase testing.

According to the standard, it is not necessary to confirm from all successive enrichment steps if pathogenic Y. enterocolitica from an earlier step was already confirmed. However, during the interlaboratory study, the laboratories agreed to confirm all the steps separately to obtain results for the evaluation of necessity of each step in the method.

Laboratories were allowed to use commercially available dehydrated culture media, ready-to-use culture media or preparation from separate ingredients. For enrichment and plating media, majority of the laboratories (10/14) prepared PSB from separate ingredients, and four laboratories used dehydrated medium Peptone Sorbitol Bile Broth (Fluka / Sigma-Aldrich). The majority of the laboratories (12/14) used dehydrated ITC broth base and respective supplements from various manufacturers (Fluka / Sigma-Aldrich, 
ten laboratories; HiMedia, two laboratories; Merck, one laboratory). Two laboratories prepared ITC from separate ingredients. For CIN agar, nine laboratories used dehydrated agar base and supplements (Oxoid, seven laboratories; Merck, four laboratories; LabM, three laboratories). Four laboratories used ready-touse CIN agar (Oxoid, three laboratories; Tammer-Tutkan maljat Oy, two laboratories).

In addition, laboratories had the opportunity to voluntarily use chromogenic media in parallel to CIN plating. YECA agar (bioMérieux, Marcy l'Etoile, France) (Denis et al. 2011) was used by ten laboratories during the study round with minced meat, and CHROMagar ${ }^{\mathrm{TM}}$ Y. enterocolitica (CHROMagar, Paris, France) (Renaud et al. 2013) was used by seven laboratories during the study rounds with raw milk, minced meat, and lettuce. In each ILS round, one enrichment was decided for the plating onto chromogenic agars beforehand to reduce the workload.

During the ILS study, three laboratories tested cold enrichment procedure as described in EN ISO 10273 Annex D (Anonymous, 2017). The cold enrichment procedure included:

a) Direct culture on CIN, sample enrichment in PSB or CEB (cold enrichment broth) at $4{ }^{\circ} \mathrm{C}$ for altogether $22 \pm 1$ days.

b) From the enrichment, after $8 \pm 1$ and $22 \pm 1 \mathrm{~d}$ of incubation, inoculation of MRB broth (modified Rappaport broth with magnesium chloride), incubation at $25^{\circ} \mathrm{C}$ for $4 \mathrm{~d}$ and plating on CIN.

c) From the enrichment, after $14 \pm 1$ and $22 \pm 1 \mathrm{~d}$ of incubation, plating on $\mathrm{CIN}$ with and without $\mathrm{KOH}$ treatment.

d) Incubation of $\mathrm{CIN}$ plates at $30^{\circ} \mathrm{C}$ for $24 \mathrm{~h}$. Identification of typical colonies and confirmation according to EN ISO 10273 (see 2.2; c and d).

\subsection{Preparation of the test materials}

The food matrix in the first ILS round in May 2013 was unpasteurized (raw) cow's milk, collected from the tank of Helsinki University farm, Finland. The matrix for the second ILS round in November 2013 was atmosphere-packed minced meat (beef-pork, 50-50\%) originating from Finland. In the third ILS round in February 2014, green crispy lettuce originating from Finland was used. All matrices were divided into portions of $25 \mathrm{ml}$ or $25 \mathrm{~g}$, and stored at $-70^{\circ} \mathrm{C}$ until use. Intrinsic background microbiota of the matrices was analysed by colony count at $30^{\circ} \mathrm{C}$ (EN ISO $4833: 2013$ ) and by enumeration of Enterobacteriaceae (EN ISO 21528-2:2004). The mean aerobic plate counts were $1.1 \times 10^{3} \mathrm{CFU} / \mathrm{ml}$ for milk, $9.8 \times 10^{3}$ for minced meat, and $1.1 \times 10^{5} \mathrm{CFU} / \mathrm{g}$ for lettuce. The mean Enterobacteriaceae counts were $<10 \mathrm{CFU} / \mathrm{ml}$ for milk, $10 \mathrm{CFU} / \mathrm{g}$ for minced meat, and $4.2 \times 10^{3} \mathrm{CFU} / \mathrm{g}$ for lettuce. The absence of pathogenic $Y$. enterocolitica in all matrices was confirmed by real-time PCR ISO/TS 18867 targeting the ail-gene (Thisted Lambertz et al. 2008, Anonymous 2015). 
1 The strain used for the inoculation of raw milk samples was $Y$. enterocolitica bioserotype 4/0:3 (Evira 595),

2 isolated from pork at Evira, Finland, in 2010. For minced meat samples, Y. enterocolitica bioserotype 2/O:9

3 (Evira 663) strain isolated from a pig carcass swab in Belgium, 2011 was used by the courtesy of Prof. Lieven

4 De Zutter, Ghent University, Belgium. Lettuce samples were inoculated with Y. enterocolitica bioserotype

5 2/O:9 strain (Evira 589), isolated from human faeces during a suspected carrot-borne outbreak in Finland, 2010, used by the courtesy of Prof. Anja Siitonen, National Institute for Health and Welfare, Finland. All the strains used for inoculation were confirmed to contain the ail-gene by real-time PCR (Thisted Lambertz et al. 2008) and virulence plasmid pYV by CR-MOX test (Riley and Toma 1989). The strains represented the pathogenic bioserotypes of $Y$. enterocolitica (4/0:3 and 2/O:9) that are the most prevalent in many parts of the world (Bottone, 1999).

For preparation of the inoculum, each ILS strain (Evira 595, 663 and 589) was streaked onto blood agar and incubated overnight at $30^{\circ} \mathrm{C}$. One colony was transferred into TSB broth (BBL ${ }^{\mathrm{TM}}$ Trypticase $^{\mathrm{TM}}$ Soy Broth, $\mathrm{BD}^{\mathrm{TM}}$, Thermo Fisher Scientific, Becton, Dickinson and Company, 38800 Le Point de Claix, France) and incubated at $30^{\circ} \mathrm{C}$ for $24 \mathrm{~h} \pm 3 \mathrm{~h}$. The incubated broth culture was serially diluted and the last dilution was made in $20 \%$ skim milk solution. Aliquots of skim milk suspension were stored at $-70^{\circ} \mathrm{C}$ until used for inoculation. From the same aliquots of skim milk, three parallel platings onto blood agar were performed to determine the concentration of the bacteria in the aliquots at the time of freezing.

For the inoculation of the samples, the skim milk aliquots were thawed at room temperature for $30 \mathrm{~min}$. Subsequently, $100 \mu \mathrm{l}$, containing the defined number of bacteria for low and high contamination level (see Table 1), were added onto each frozen sample. Inoculation at low level was designed to facilitate the calculation of the level of detection $\left(\mathrm{LOD}_{50}\right)$ by returning fractional positive results (not every sample positive or negative). For the determination of the number of inoculated bacteria, $100 \mu$ aliquots of skim milk suspension were plated onto four parallel blood agar plates at regular intervals during the inoculation of samples ( 8 to 11 times, resulting in a total of 32 to 44 plates on each level). After the inoculation, the samples were immediately transferred back to $-70^{\circ} \mathrm{C}$ for storage until the time of dispatch. All plates were incubated for $44 \pm 4 \mathrm{~h}$ at $30^{\circ} \mathrm{C}$, and the number of inoculated bacteria at each level was calculated as the mean value of all the counts obtained. The blank samples were prepared separately (on a different day) from inoculated samples by adding sterile skim milk on frozen samples. The samples were sealed tightly and handled separately until packing for transportation with other samples. 
For homogeneity studies, analyses of 60 samples (20 samples from each contamination level: blank, low and high) were started 13 (raw milk study), 6 (minced meat study) or 8 days (lettuce study) before the start of each ILS round. The analyses for homogeneity were conducted according to the ILS laboratory procedures. In addition, the homogeneity of the prepared inoculum was assessed from the colony counts determined when inoculating the samples (see 2.3).

For stability studies, 60 samples of each round (20 samples from each level: blank, low and high) were divided into two sets of 30 samples which were stored under two different conditions representing optimal transport conditions (Stability study 1, 10 samples from each level) and suboptimal transport and storage conditions (Stability study 2, 10 samples from each level). To mimic optimal transport conditions, the samples were packed in dry ice and the transport packages were kept at room temperature $\left(22\right.$ to $\left.25^{\circ} \mathrm{C}\right)$ for 20 to $24 \mathrm{~h}$ before the start of the analysis. To mimic suboptimal transport conditions, the samples were packed in dry ice and the transport packages were kept at room temperature for three days, after which the packages were opened and the samples were transferred for storage at $-20^{\circ} \mathrm{C}$ for three or four days before the start of the analysis.

Additionally, the stability of the strain suspension that was used for inoculation was verified towards successive thawing and re-freezing by colony count (as described in 2.3) before each trial round. This was to ensure that the number of bacteria remained stable during the additional freezing in the inoculation process (see 2.3).

\subsection{Statistical analysis of the data}

The plate counts of inocula for homogeneity assessment were analysed according to ISO/TS 22117:2010 Annex $B, T_{1}-T_{2}$ test and acceptable variation between units of a batch were considered to be $T 2 /(I-1) \leq$ 2.

The $\mathrm{LOD}_{50}$ was calculated using PODLOD calculation program version 5 (Wilrich and Wilrich, 2009, ISO 16140-2:2015, http://www.wiwiss.fu-berlin.de/fachbereich/vwl/iso/ehemalige/wilrich/index.html, accessed 14.3.2017)

A logistic regression model (M1) was applied to evaluate the necessity of parallel enrichment steps and alkaline $(\mathrm{KOH})$ treatment in the laboratory procedure. The effects of ten complementary options for the method (T2-T11, Table 4), later referred to as treatments, on the sensitivity of detection were compared with relation to the reference; direct plating of PSB initial suspension on CIN agar (without enrichment) (T1). The variation of detection that arose from the experimental setting was considered in the model by including fixed effects for the contamination level and ILS, and random effect for the laboratories. 
logit $\left(\theta_{i}\right)=\mu_{0}+$

1

$$
\begin{aligned}
& \alpha_{1} \cdot T 1_{i}+\alpha_{2} \cdot T 2_{i}+\alpha_{3} \cdot T 3_{i}+\alpha_{4} \cdot T 4_{i}+\alpha_{5} \cdot T 5_{i}+ \\
& \beta\left[\text { Level }_{i}\right]+\gamma\left[I L S_{i}\right]+\delta\left[L a b_{i}\right]
\end{aligned}
$$

$2 y_{i} \mid \theta_{i} \sim \operatorname{Bin}\left(n_{i}, \theta_{i}\right)$,

3 in which

4

5

6

7

8

9

10

11

$y_{i}$ is the number of true positive samples detected per contamination level per ILS per laboratory

$n_{i}$ is the number of true positive samples per contamination level per ILS per laboratory $\theta_{i}$ is the proportion of true positive samples detected (or sensitivity) per contamination level per ILS per laboratory

with uninformative prior probability densities

$$
\begin{aligned}
& p\left(\mu_{0}\right)=N\left(0,10^{3}\right) \\
& p\left(\alpha_{j}\right)=N\left(0,10^{3}\right), \quad j=1, \ldots, 5 \\
& p\left(\beta_{k}\right)=N\left(0,10^{3}\right), \quad k=1,2 \\
& p\left(\gamma_{l}\right)=N\left(0,10^{3}\right), \quad l=1,2,3 \\
& p\left(\delta_{m}\right)=N\left(0, \tau^{-1}\right), \quad m=1, \ldots, 14 \\
& p(\tau)=\operatorname{Gamma}\left(10^{-3}, 10^{-3}\right)
\end{aligned}
$$

Detection probabilities and odds ratios (ORs, relative to $T 1$ ) for the single treatments $T 2-T 5$ were defined with equations (E1) and (E2). These were further used for calculating detection probabilities and odds ratios for the combined treatments T6-T11 with equations (E3) and (E4).

$\operatorname{logit}(p(T j))=\mu_{0}+\alpha_{j}, \quad j \in\{1, \ldots, 5\}, \quad \alpha_{1}=0 \quad$ for the reference $\quad T 1$

$$
\mathrm{OR}(T j)=e^{\alpha_{j}}, \quad j \in\{2, \ldots, 5\}
$$

$p(T B)=1-\prod_{r=1}^{R}\left[1-p\left(T A_{r}\right)\right], \quad T B \in\{T 6, \ldots, T 11\}, \quad T A_{r} \in\{T 1, \ldots, T 5\}$

$$
\mathrm{OR}(T B)=\frac{p(T B) /(1-p(T B))}{p(T 1) /(1-p(T 1))}, \quad T B \in\{T 6, \ldots, T 11\}
$$

7 Data were analysed by using the R software (version 3.3.2; R Core Team [https://www.R-project.org/]) and 8 OpenBUGS software (version 3.2.3 rev 1012; OpenBUGS Project Management Group 
[http://www.openbugs.net/]) via R2OpenBUGS package. Dataset contained only the observations from treatments T1-T5 on low and high contamination level, and observations with missing values were removed before the analysis. Markov Chain Monte Carlo simulations were run in one chain with 10000 iterations after adaptation of 2,000 iterations. For each OR, mean and $95 \%$ posterior credibility interval were calculated from 10000 iterations. If $95 \%$ interval for $O R>1.0$, treatment was interpreted to increase the sensitivity of detection with relation to the reference. Differences in effects between two treatments were reported if their $95 \%$ intervals for ORs appeared non-overlapping. In addition to the treatment effects over all ILS rounds, datasets were formed and analysed for each ILS separately to observe any variation between food matrices or strains. After evaluating the results, analyses were repeated by setting T2 (PSB enrichment with $\mathrm{KOH}$ ) as the reference. Datasets and codes for $\mathrm{R}$ and OpenBUGS are available from the authors upon request.

\section{Results and discussion}

\subsection{Homogeneity and stability of the test materials}

In homogeneity testing, the inoculated raw milk, minced meat and lettuce samples gave the expected numbers of positive results. Furthermore, the plate counts determined during preparation of the inocula were within the acceptable variation at the batch level in each study round (Table S1). The mean counts for low and high inoculum level were respectively 9 CFU (standard deviation, SD 3.0 CFU) and 59 CFU (SD 7.7 CFU) for raw milk; 16 CFU (SD 4.0 CFU) and 85 CFU (SD 15 CFU) for minced meat; and 110 CFU (SD 8.6 CFU) and $1100 \mathrm{CFU}$ ( SD $11 \mathrm{CFU}$ ) for lettuce. Thus, the samples and the inocula were considered homogenous. Likewise, with expected number of positive results, the samples were considered stable, even under suboptimal shipping conditions (Table S1).

\subsection{General results of the study}

The majority of the laboratories received their samples within $27 \mathrm{~h}$ after dispatch and could directly proceed to analysis or storage of the samples. Exceptionally, three laboratories in the study round with minced meat and one laboratory in the study round with lettuce received their samples two days after dispatch. They stored the samples at $-20^{\circ} \mathrm{C}$ or $-70^{\circ} \mathrm{C}$ and started the analysis seven days after dispatch. As indicated by the temperature logger data and individual temperature measurements by the laboratories, the temperature was stable during shipping. Moreover, the samples were verified to be stable for altogether seven days (see 3.1). 
1 After evaluation of deviations, results of two laboratories in the study round with raw milk were excluded

2 from further analysis based on deviation from the protocol. For one laboratory in the study round with

3 lettuce, one blank sample and one sample with the low inoculation level were excluded from the analysis

4 due to lack of confirmation tests. The results of the other laboratories could be included after detailed examination and requests of further information, to ensure that there were no deviations from the protocol.

7

Summary of participant laboratories' positive sample results at each inoculation level and sample type is given in Table 1.

\subsection{Analysis of the data}

\subsubsection{Specificity}

The laboratories examined eight blank samples per food type, a total of 24 samples expected to give negative results. In ILS round with raw milk, no false positive results were observed. In ILS round with minced meat, three laboratories reported false positive results (Table 1); one laboratory with two out of eight samples and two laboratories with one sample out of eight. Six isolates recovered by two laboratories from a blank sample were sent to the organizing laboratory for further analysis. In ILS round with lettuce, two laboratories reported one false positive result each. The isolates recovered were available for further characterization from one of the laboratories. All the isolates were indistinguishable from the strains used for inoculation of the samples, based on the results obtained from biochemical tests and biotyping according to ISO 10273, ail-gene detection by ISO/TS 18867 and PFGE analysis (data not shown). This suggests cross-contamination of the samples, either by the organizer or by the participating laboratories, but the site of the cross-contamination could not be verified. Every precaution was taken at the organizing laboratory not to cross-contaminate the samples. Neither did the participants report leakage of the samples during shipment. The specificity of the method was $100 \%$ with raw milk (Table 2), $96 \%$ with minced meat (Table 3) and $98 \%$ with lettuce samples (Table 4).

\subsubsection{Sensitivity}

The overall sensitivity of the method at the high contamination level was $96 \%$ with raw milk (Table 2), $97 \%$ with minced meat (Table 3) and 98\% with lettuce samples (Table 4). Based on the study design, a sensitivity around $50 \%$ was expected for the low level. The sensitivities at the low inoculation level were $68 \%$ for raw milk, $77 \%$ for minced meat, and $81 \%$ for lettuce. 
2 Participating laboratories agreed to confirm separately all the parallel steps of the method leading to

3 isolation (see 2.2 for the explanation of the procedure), which made it possible to compare the necessity of parallel steps for the general outcome of the procedure. Participant laboratories' positive results and samples analysed at each enrichment and plating step of the method are summarized in Table 5.

The sensitivity of direct culture varied from $2 \%$ to $21 \%$ in raw milk and minced meat with the low and high inoculation level and in lettuce matrix with the low inoculation level, whereas the sensitivity increased to $64 \%$ in lettuce samples with the high inoculation level (Table 5). Despite of this surprisingly high recovery of pathogenic $Y$. enterocolitica in lettuce matrix without any enrichment, direct culture alone was insufficient for optimal recovery in all three matrices. However, direct culture was added in the revised method as mandatory due to its capacity to give rapid results in certain cases of food sample analysis.

The sensitivity of PSB enrichment step in different sample types varied between $44 \%$ and $90 \%$ when $\mathrm{KOH}$ treatment was used before plating, and between $3 \%$ and $24 \%$ in the absence of $\mathrm{KOH}$ treatment. When except for lettuce samples, where enrichment in ITC and $\mathrm{KOH}$ treatment before plating on CIN showed the highest sensitivity $82 \%$ (high inoculation level). In comparison, the sensitivity of PSB and $\mathrm{KOH}$ was $74 \%$ (high inoculation level) in lettuce. In raw milk and minced meat samples, the sensitivity of PSB with $\mathrm{KOH}$ treatment varied between $52 \%$ and $90 \%$. The sensitivity of ITC varied between $15 \%$ and $82 \%$ with $\mathrm{KOH}$ treatment before plating, and between $7 \%$ and $60 \%$ without $\mathrm{KOH}$ treatment, in all three matrices.

\subsubsection{Statistical analysis for the effect of isolation steps on sensitivity}

Of the single treatments (T2-T5), the highest OR (95\% interval: 9.4-15.3) was observed for T2 (PSB with $\mathrm{KOH}$ ) with relation to the reference $T 1$ (direct culture) in the analysis over all ILS rounds. Furthermore, treatment $T 2$ increased the sensitivity of detection more than T4 (ITC with $\mathrm{KOH}$; OR 3.3-5.2), and thus, higher performance was observed for PSB than for ITC enrichment. For both PSB and ITC, higher performance was observed with $\mathrm{KOH}$ treatment than without it. Without $\mathrm{KOH}$ treatment, ORs appeared respectively $0.3-0.5(T 3)$ and 1.2-2.0 (T5). This suggests that the isolation step without $\mathrm{KOH}$ was unnecessary and could be omitted from the method.

Because the highest performance was observed for $T 2$ with relation to the reference $T 1$, treatment $T 2$ was set as the new reference to further evaluate the necessity of parallel steps in the method. In the analysis over all ILS rounds, treatment T8 (parallel enrichment in PSB and ITC, both with $\mathrm{KOH}$ treatment) was 
observed to increase the sensitivity of detection (OR 1.6-2.0) with relation to the reference T2 (PSB with $\mathrm{KOH})$ (Table 6). Odds ratios of similar order were observed for $T 10$ and $T 11$, respectively 1.9-2.4 and 2.33.3, suggesting that the sensitivity of detection cannot be increased significantly by adding direct culture and steps without $\mathrm{KOH}$ in the method. However, direct culture was agreed to be a mandatory part of the procedure already during the revision because of its advantage to produce rapid results with certain sample types. Therefore, $T 10$ was chosen as the mandatory procedure in the method, containing direct culture in addition to parallel enrichment in PSB and ITC, plating both with $\mathrm{KOH}$ treatment.

When ILS rounds were analysed separately, the performance of the enrichment broths were observed to vary between the food matrices (or strains). In the ILS rounds for raw milk and minced meat, lower performance was observed for ITC (T4) compared with the reference, PSB (T2). In contrast, higher performance (OR 1.4-3.0) was observed for ITC (T4) in the ILS round for lettuce. This further supports parallel inclusion of PSB and ITC in the standard method. Apparently, ITC successfully suppressed the background microbiota of lettuce matrix and therefore compensated the performance of non-selective PSB in detection of pathogenic $Y$. enterocolitica. Omitting steps without $\mathrm{KOH}$ from the method was also supported by the separate analyses of the ILS rounds for minced meat and lettuce, which provided no evidence on the difference in performance between steps with $\mathrm{KOH}$ treatment $(T 10)$ and when supplemented with steps without $\mathrm{KOH}$ (T11). For raw milk, however, the inclusion of step without $\mathrm{KOH}$ was beneficial: slightly higher OR (2.4-3.9) was observed for T11 than for T10 (1.6-2.3). As the data revealed, three laboratories detected positive samples at low level (one, two, and three samples positive out of eight, respectively) from PSB without $\mathrm{KOH}$ that remained negative with other treatments. This supports the optional inclusion of step without $\mathrm{KOH}$ in the method, which can be advantageous with certain sample types.

Noteworthy, the better performance of $\mathrm{KOH}$ treatment depends on the success of the treatment. This is mostly affected by the preparation and shelf life of $\mathrm{KOH}$, specified as "preparation the day before use" in the standard revision. The better performance of PSB compared to ITC, in turn, could be explained by the inoculum size; the initial suspension of ITC is prepared by transferring $10 \mathrm{ml}$ of initial suspension in PSB into $90 \mathrm{ml}$ of ITC and thus resulting in smaller fraction of the bacteria in enrichment than with PSB. For certainty of transferring at least one microorganism (Yersinia cell, in this case) in $1 \mathrm{ml}$, the suspension requires average level of at least seven cells per $\mathrm{ml}$ of the culture (Jarvis, 2007). Thus, the lower recovery when using ITC compared with PSB enrichment may have been purely by chance of not transferring any of the inoculated cells into ITC at the low inoculation level. This could have affected isolation especially from milk samples, in which the inoculum was the lowest. Along with fewer cells transferred to ITC, selective ingredients of ITC could inhibit also the growth of $Y$. enterocolitica, resulting in PSB to surpass ITC. 
Especially, this could affect isolation from sample matrices in which the selective properties of ITC are dispensable or isolation of strains that are more susceptible. De Zutter et al., 1994 reported that growth of serotype 0:9 in ITC was supressed in comparison to serotype 0:3 in pork samples, especially due to potassium chlorate of ITC.

\subsubsection{Level of detection $\operatorname{LOD}_{50}$}

The low inoculation level included in the ILS was designed to give fractional positive results making it possible to assess $\mathrm{LOD}_{50}$ of the method, meaning the specific concentration of pathogenic $Y$. enterocolitica that gives $50 \%$ probability of detection. Values of $L D_{50}$ varied depending on the combination of the matrix and strain studied. In raw milk inoculated with Y. enterocolitica bioserotype 4/O:3 strain, LOD $_{50}$ was 9.4 $\mathrm{CFU} / 25 \mathrm{ml}$. In minced meat and lettuce, each inoculated with different $Y$. enterocolitica bioserotype 2/O:9 strain, $\mathrm{LOD}_{50}$ was $9.9 \mathrm{CFU} / 25 \mathrm{~g}$ and $63 \mathrm{CFU} / 25 \mathrm{~g}$, respectively (Tables 2-4). The relatively higher $\mathrm{LOD}_{50}$ in lettuce may have resulted from the presence of higher background microbiota, such as Enterobacteriaceae, which may have adverse effect on the level of detection. Because the enrichment and plating media used in the method are not highly selective for pathogenic $Y$. enterocolitica, other members of Enterobacteriaceae family, non-pathogenic Yersinia and even non-pathogenic environmental Y. enterocolitica can easily overgrow pathogenic $Y$. enterocolitica. Furthermore, these organisms may be picked for confirmation instead of pathogenic $Y$. enterocolitica if the laboratory is not experienced with the analysis.

However, preliminary studies at the organizing laboratory showed that $\mathrm{LOD}_{50}$ varied notably depending on the strain used for inoculating the lettuce matrix (data not shown). This suggests that the pathogenic $Y$. enterocolitica strain has a major role in the probability of detection, besides the background microbiota and the composition of food. Eventually, no single isolation procedure appears to be absolute for the recovery of all human-pathogenic Y. enterocolitica strains in foods (De Boer, 2003; Fredriksson-Ahomaa and Korkeala 2003).

The effects on $\mathrm{LOD}_{50}$ using different enrichment and plating steps and the combinations of them are shown in Table 7. With direct culture as the only step in the method, $\operatorname{LOD}_{50}$ values were approximately ten times higher as compared to the whole protocol. The whole protocol consisted of direct plating on CIN agar, parallel enrichment in PSB and ITC, both followed by parallel plating onto CIN with and without $\mathrm{KOH}$ treatment. By performing only the direct plating and PSB with $\mathrm{KOH}, \mathrm{LOD}_{50}$ values approached those achieved by performing the whole protocol with raw milk and minced meat, but not with lettuce. With lettuce samples, $\mathrm{LOD}_{50}$ remained approximately ten times higher unless a combination of PSB and ITC was used. Steps that were selected as mandatory in the revised EN ISO 10273 resulted in LOD 50 values of similar 
order (12 CFU/25 ml in raw milk, $11 \mathrm{CFU} / 25 \mathrm{~g}$ in minced meat and $68 \mathrm{CFU} / 25 \mathrm{~g}$ in lettuce) when compared with the whole protocol. Mandatory steps of the revised standard differed from the whole protocol by exclusion of the plating without $\mathrm{KOH}$ step after PSB and ITC enrichment.

\subsubsection{Use of chromogenic media}

Only five laboratories included chromogenic media in their test procedure in all three ILS rounds. To increase the comparability of the results, evaluation was based on seven laboratories that used chromogenic media in both rounds with minced meat and lettuce. In each round, one enrichment for the plating onto chromogenic agars was decided beforehand. The results are summarized in Table S2 where comparison to the respective (parallel) CIN plating step can be made. Choosing the enrichment broth beforehand aimed to reduce the workload of laboratories but did not always produce the maximum number of presumptive positive colonies for confirmation. For example, in ILS round with minced meat, ITC was chosen beforehand as enrichment for the plating onto chromogenic agars, but PSB appeared to be more sensitive enrichment in that round. The sensitivity of chromogenic media was usually lower than or equal to CIN (Table S2). However, the sensitivity was equal or superior to CIN in the low and high inoculation level lettuce samples, respectively, when plated from PSB with $\mathrm{KOH}$ treatment. Overall, the limited number of results showed high variation. For example, one laboratory reported 16 positives out of 16 samples and found the chromogenic agar beneficial, while three laboratories reported no positives and other three laboratories reported one to three samples positive from the corresponding minced meat samples (data not shown). The usefulness of chromogenic media to the overall sensitivity of detection seemed to be limited. Only three laboratories reported single correct positive results by using chromogenic agars while parallel $\mathrm{CIN}$ agar plates remained negative, in minced meat and lettuce samples. The results for chromogenic agars are only indicative and should be interpreted with caution. The study resulted not enough data to justify the mandatory use of chromogenic media as primary plating media after enrichment, but supported its optional use in the method.

\subsubsection{Confirmation of the results in ISO 10273}

In the revised standard method ISO 10273 (Anonymous 2017), suspected colonies from primary culture are streaked (purified) on CIN agar and (optionally) on chromogenic agar to facilitate selection of characteristic colonies for further confirmation. The selection based on colony morphology (Figure S2) is done by using a stereomicroscope which is emphasized in the revised standard method. Microscoping can markedly reduce the number of isolates needing further confirmation and thus decrease the workload. During the ILS, chromogenic media performed especially well in distinguishing between colonies of potentially pathogenic 
and non-pathogenic Yersinia when choosing isolates for confirmation. The same can also be achieved by using CIN (Hallanvuo et al., 2006) but this requires more expertise on colony morphology than simple colour difference detection in chromogenic media. However, when intrinsic sample microbiota in ILS study contained non-pathogenic $Y$. enterocolitica -like species $Y$. mollaretii and/or $Y$. bercovieri, their colonies could not be differentiated from pathogenic $Y$. enterocolitica based on colour on chromogenic media. In these cases, colony morphology by stereomicroscope again revealed differences (data not shown).

During the interlaboratory study rounds, seven to eight voluntary laboratories confirmed the results by using two pathways (see Figure S1 in supplementary material). Laboratories used real-time PCR for the detection of pathogenic Y. enterocolitica as described in ISO/TS 18867 (Anonymous 2015), Annex B, Methods 1 or 2 together with pyrazinamidase testing as described in ISO 10273, for parallel comparison to biochemical confirmation pathway of ISO 10273 (Anonymous 2017). Altogether 410 parallel confirmation reactions for 319 samples representing all study rounds gave concordant results (Table S3), thus validating the use of real-time PCR ISO/TS 18867 together with pyrazinamidase testing, in confirmation of pathogenic Y. enterocolitica according to ISO 10273. The option of using two alternative pathways in confirmation (see Figure S1) was therefore included in the standard revision (Anonymous 2017).

In the context of standard ISO 10273, pyrazinamidase is an important mandatory test related to virulence of $Y$. enterocolitica (Farmer et al., 1992). It was noted, however, that this test is prone to errors related to the amount of inoculum and the preparation and shelf life of ammonium iron (II) sulphate solution used in the test. Notably, the possibility of obtaining false negative results increases if limited amount of inoculum is used. Conversely, the possibility of obtaining false positive reactions increases if old ammonium iron (II) sulfate solution is used. Therefore, the amount of inoculum (generous loopful of bacteria) and the shelf-life

\subsubsection{Cold enrichment procedure}

Comparison of the performance of PSB and CEB broths by one laboratory (vegetable samples) together with the organizing laboratory (raw milk and minced meat samples) allowed the choice of using either PSB 
or CEB in the cold enrichment procedure of EN ISO 10273, Annex D (data not shown). During the ILS study with lettuce samples, two laboratories tested the cold enrichment procedure and reported 15 samples positive out of 16 , and 11 samples positive out of 16 , respectively, by using PSB enrichment at $4^{\circ} \mathrm{C}$. During the same study round, one of these laboratories also used CEB enrichment at $4^{\circ} \mathrm{C}$ and reported 16 samples positive out of 16 . Both laboratories reported 14 samples positive out of 16 with the ILS study protocol (direct culture, PSB and ITC, see 2.2). Therefore, the cold enrichment procedure performed equal to the ILS study protocol, although this observation was based on limited data obtained during the study. As the incubation time in cold enrichment is extremely long, up to 23 days, this procedure was considered inconvenient for general use. However, it was retained in the informative Annex D of EN ISO 10273 to be used, for example, during outbreak investigations when additional procedures may be needed.

\subsection{Quality assurance of the culture media}

The requirement for performance testing for the quality assurance of the culture media used in the microbiological methods is universal in standardization. The productivity of ITC, PSB, CIN and nutrient agar media, and the selectivity of ITC shall be tested with the control strains and scheme specified in revised standard EN ISO 10273 Annex B (Anonymous 2017). Before the revision, the specifications were only described in ISO 11133 (Anonymous 2014) and were to be transferred in EN ISO 10273. However, during testing of the criteria in the organizing laboratory, the need for changing testing scheme and control strains was noted. The scheme was reorganised according to standard ISO 11133 Annex J instructions. The revised scheme was tested and verified by two participating independent ILS laboratories; for ITC and PSB each, two producers and four lots of media were tested, and for CIN, four producers and seven lots were tested (data not shown). As the scheme passed the testing, it was included in the standard revision (Anonymous 2017).

\section{Conclusions}

Performance parameters for international standard EN ISO 10273 were established in an interlaboratory study carried out by 14 laboratories in response to Mandate M/381 by European Commission. The level of detection, $\mathrm{LOD}_{50}$, of the method was $9.4 \mathrm{CFU} / 25 \mathrm{ml}$ in raw milk, $9.9 \mathrm{CFU} / 25 \mathrm{~g}$ in minced meat and 63 CFU/25 g in lettuce samples. The method showed sensitivity of $96 \%, 97 \%$, and $98 \%$ in raw milk, minced meat, and lettuce samples, respectively, at inoculation levels of pathogenic $Y$. enterocolitica 5-10 times above the level of detection. The specificity was $100 \%$ in raw milk, $96 \%$ in minced meat, and $98 \%$ in lettuce. 
Confirmation of colonies by real-time PCR method ISO/TS 18867 (Anonymous 2015) together with pyrazinamidase testing produced $100 \%$ concordant results with conventional biochemical confirmation. Thus, ISO/TS 18867 and pyrazinamidase testing can be used as an alternative confirmation procedure to biochemical confirmation. As every parallel step leading to the isolation of pathogenic $Y$. enterocolitica was confirmed during the trial, it was possible to evaluate the performance and necessity of parallel steps of the method. PSB enrichment and plating on $\mathrm{CIN}$ by using alkaline $(\mathrm{KOH})$ treatment showed the highest sensitivity (52-92\%, both levels of inoculation) in raw milk and minced meat samples. This was not evident in lettuce samples, in which ITC enrichment followed by $\mathrm{KOH}$ treatment before plating on CIN showed higher sensitivity (64\% at low and $82 \%$ at high inoculation level) than plating on CIN from PSB with $\mathrm{KOH}$ treatment (44\% at low and $74 \%$ at high inoculation level). The necessity of both enrichment broths, PSB and ITC, was supported by the statistical analysis. However, this analysis indicated no difference in the performance if steps without $\mathrm{KOH}$ were excluded from the ILS study protocol. Based on the analysis, plating on the $\mathrm{CIN}$ agar without $\mathrm{KOH}$ treatment could be left as optional procedure in the method.

\section{Acknowledgements}

The validation of international standard EN ISO 10273 has been carried out under the framework of European Mandate No. M381 of DG SANTE and DG GROW (European Commission).

The authors wish to thank the coordination team of this Mandate, from CEN/TC275/WG6 "Microbiology of the food chain" Alexandre Leclercq (convenor), Gwenola Hardouin (secretary), from ISO/TC34/SC9 "Food products - Microbiology" Bertrand Lombard (chairman, convenor of WG2 "Statistics"), Paul in't Veld (convenor of WG3 "Method validation");

-The experts of the working group CEN/TC275/WG6 TAG 20

-Thorsten Trumpf, IVUA Lebensmittel-, Veterinär- und Umweltuntersuchungsamt des Landes SchleswigHolstein, Max-Eyth-Str. 524537 Neumünster, Germany; Helena Koskentalo, and Seija Raivio, SeiLab Vaasantie 1 C, 60100 Seinäjoki, Finland; Kaisa Aro, Water Protection Association of the River Kokemäki, Lensunkatu 9, 26100 Rauma, Finland; Eeva Klemettilä-Kirjavainen, Metropolilab Oy, Viikinkaari 4, 00790 Helsinki, Finland, for taking part in the ILS.

-Flemming Hansen, Danish Meat Research Institute DMRI, Dep of Hygiene \& Processing, 2 Maglegaardsvej, DK-4000 Roskilde, Denmark, and Markus Tzschoppe and Erika Hufnagel, WESSLING GmbH, Impexstrasse 5, 69190 Walldorf, Germany, for taking part in the pre-trial.

-Anne Wilen, Elina Poutanen and Kirsi-Maria Eklund for technical assistance; 


\section{References}

Anonymous, 2003. EN ISO 10273. Microbiology of food and animal feeding stuffs - Horizontal method for the detection of presumptive pathogenic Yersinia enterocolitica. Int. Org. for Standardization, Geneva, Switzerland.

Anonymous, 2004. EN ISO 21528-2. Microbiology of food and animal feeding stuffs -- Horizontal methods for the detection and enumeration of Enterobacteriaceae -- Part 2: Colony-count method. Int. Org. for Standardization, Geneva, Switzerland.

Anonymous, 2010. CEN ISO/TS 22117, Microbiology of food and animal feeding stuffs - Specific requirements and guidance for proficiency testing by interlaboratory comparison. Int. Org. for Standardization, Geneva, Switzerland.

Anonymous, 2013. EN ISO 4833. Microbiology of the food chain - Horizontal method for the enumeration of microorganisms - Part 2: Colony count at $30^{\circ} \mathrm{C}$ by the surface plating technique. Int. Org. for Standardization, Geneva, Switzerland.

Anonymous, 2015. ISO/TS 18867:2015, Microbiology of the food chain - Polymerase chain reaction (PCR) for the detection of food-borne pathogens - Detection of pathogenic Yersinia enterocolitica and Yersinia pseudotuberculosis. Int. Org. for Standardization, Geneva, Switzerland.

Anonymous, 2017. EN ISO 10273. Microbiology of the food chain - Horizontal method for the detection of pathogenic Yersinia enterocolitica. Int. Org. for Standardization, Geneva, Switzerland.

Bottone, E.J. 1999. Yersinia enterocolitica: Overview and epidemiologic correlates. Microbes Infect. 1 (4), 323-333.

De Boer, E. 2003. Isolation of Yersinia enterocolitica in foods. In: Corry, J.E.L., Curtis, G.D.W. and Baird, R.M. "Handbook of Culture Media for Food Microbiology", Elsevier, p. 215-228.

Denis, M., Houard, E., Labbé, A., Fondrevez, M., Salvat, G.A. 2011. Selective chromogenic plate, YECA, for the detection of pathogenic Yersinia enterocolitica: specificity, sensitivity, and capacity to detect pathogenic Y. enterocolitica from pig tonsils. Journal of Pathogens, Article ID 296275, 8 pages, 2011. doi:10.4061/2011/296275

De Zutter, L., Le Mort, L., Janssens, M., Wauters, G. 1994. Short-comings of irgasan ticarcillin chlorate broth for the enrichment of Yersinia enterocolitica biotype 2, serotype 9 from meat. Int. J. Food Microbiol. 23, 231-237. 
EFSA European Food Safety Authority. 2009. Technical specifications for harmonised national surveys of Yersinia enterocolitica in slaughter pigs on request of EFSA. EFSA Journal 7(11), 1374. [23 pp.]. doi:10.2903/j.efsa.2009.1374.

Farmer, J.J. III, Carter, G.P., Miller, V.L., Falkow, S. and Wachsmuth, I.K. 1992. Pyrazinamidase, CR-MOX agar, salicin fermentation - esculin Hydrolysis, and D-xylose fermentation for identifying pathogenic serotypes of Yersinia enterocolitica. J. Clin. Microbiol. 30, 2589-2594.

Fredriksson-Ahomaa, M. and Korkeala, H. 2003. Low occurrence of pathogenic Yersinia enterocolitica in clinical, food, and environmental samples: a methodological problem. Clin. Microbiol. Rev. 16(2), 220-229. Hallanvuo S., Peltola J., Heiskanen T. and Siitonen A. 2006. Simplified phenotypic scheme evaluated by $16 \mathrm{~S}$ rRNA sequencing for differentiation between Yersinia enterocolitica and Y. enterocolitica-like species. J. Clin. Microbiol., 44, 1077-1080.

Jarvis, B., 2007. On the compositing of samples for qualitative microbiological testing. Lett. Appl. Microbiol. 45, 592-598.

Renaud, N., Lecci, L., Courcol, R.J., Simonet, M., and Gaillot, O. 2013. CHROMagar Yersinia, a new chromogenic agar for screening of potentially pathogenic Yersinia enterocolitica isolates in stools. J. Clin. Microbiol. 51, 1184-1187.

Riley, G. and Toma, S. 1989. Detection of pathogenic Yersinia enterocolitica by using Congo red-magnesium oxalate agar medium. J. Clin. Microbiol. 27, 213-214.

Thisted Lambertz, S, Nilsson C, Hallanvuo S, Lindblad, M. 2008. Real-time PCR method for detection of pathogenic Yersinia enterocolitica in food. Appl. Environ. Microbiol. 74, 6060-6067.

Van Damme I., Habib I., De Zutter L., 2010. Yersinia enterocolitica in slaughter pig tonsils: Enumeration and detection by enrichment versus direct plating culture. Food Microbiology 27(1), 158-61. Epub 2009 Sep 27. doi:10.1016/j.fm.2009.09.011

Van Damme, I., Berkvens, D. and De Zutter, L. 2012. Effect of sampling and short isolation methodologies on the recovery of human pathogenic Yersinia enterocolitica from pig tonsils. Foodborne Pathog. Dis. 9, 600-606. doi: 10.1089/fpd.2012.1128. Epub 2012 Jun 12.

Weagant, S.D. 2008. A new chromogenic agar medium for detection of potentially virulent Yersinia enterocolitica. J. Microbiol. Meth. 72, 185-190.

Wilrich, C. and Wilrich, P-Th. 2009. Estimation of the POD function and the LOD of a qualitative microbiological measurement method. J. AOAC Int. 92, 1783- 1792. 
Authors Affiliations:

${ }^{\mathrm{a}}$ Food and Feed Microbiology Research Unit and ${ }^{\mathrm{b}}$ Risk Assessment Research Unit, Research and Laboratory

4 Department, Finnish Food Safety Authority Evira, Mustialankatu 3, 00790 Helsinki, Finland

5 ' Interlaboratory study group: Nadine Botteldoorn, Service foodpathogens, Institute of Public health,

6 Juliette Wijtsmansstreet 14, 1050 Brussels, Belgium; Lieven De Zutter, Department of Veterinary Public

7 Health and Food Safety, Faculty of Veterinary Medicine, Ghent University, Salisburylaan, 133, 9820

8 Merelbeke, Belgium. Maria Fredriksson-Ahomaa, Faculty of Veterinary Medicine, University of Helsinki,

9 P.O. Box 66, Fl-00014 Helsinki University, Finland; Stefan Hertwig, Federal Institute for Risk Assessment

10 (BfR), Diedersdorfer Weg 1, 12277 Berlin, Germany; Gro S. Johannessen, Norwegian Veterinary Institute,

11 Ullevålsveien 68, 0454 Oslo, Norway; Martina Ludewig, Institute of Food Hygiene, Faculty of Veterinary

12 Medicine, University of Leipzig, An den Tierkliniken 1, 04103 Leipzig, Germany; Ute Messelhäußer,

13 Laboratory of food microbiology, Bavarian Health and Food Safety Authority, Veterinärstr. 2, 85764

14 Oberschleißheim, Germany; Pia Sigvart-Mattila, Water Protection Association of the River Kokemäki,

15 Patamäenkatu 24, 33900 Tampere, Finland; Susanne Thisted-Lambertz, Microbiology division, National

16 Food Agency, Strandbodgatan 4, 75323 Uppsala, Sweden; Tiina Thure, Metropolilab Oy, Viikinkaari 4, 00790

17 Helsinki, Finland; Elina Vatunen, Finnish Customs Laboratory, Tekniikantie 13, 02150 Espoo, Finland

$18{ }^{1}$ Present address: Research Services, P.O. Box 62, FI-00014 University of Helsinki, Finland

$19 *$ Corresponding author (project leader).E-mail address: saija.hallanvuo @ evira.fi 
Tables:

Table 1. Participant laboratories' positive sample results (the maximum number of results is eight per inoculation level).

\begin{tabular}{|c|c|c|c|c|c|c|c|c|c|c|c|c|c|c|c|}
\hline \multirow{2}{*}{$\begin{array}{l}\text { Sample } \\
\text { matrix }\end{array}$} & \multirow{2}{*}{$\begin{array}{l}\text { Inoculation } \\
\text { level }^{\mathrm{a}}\end{array}$} & \multicolumn{14}{|c|}{ Laboratory } \\
\hline & & LAB 23 & LAB 24 & LAB 25 & LAB 26 & LAB 27 & LAB 28 & LAB 29 & LAB 31 & LAB 32 & LAB 34 & LAB 35 & LAB 36 & LAB 37 & LAB 39 \\
\hline \multirow{3}{*}{$\begin{array}{l}\text { Raw } \\
\text { milk }\end{array}$} & Blank & 0 & 0 & 0 & ND & 0 & 0 & 0 & 0 & 0 & 0 & 0 & 0 & ND & 0 \\
\hline & Low & 5 & 7 & 3 & ND & 8 & 8 & 5 & 5 & 6 & 7 & 4 & 3 & ND & 4 \\
\hline & High & 8 & 8 & 5 & ND & 8 & 8 & 8 & 8 & 8 & 8 & 6 & 6 & ND & 8 \\
\hline \multirow{3}{*}{$\begin{array}{l}\text { Minced } \\
\text { meat }\end{array}$} & Blank & 0 & ND & $2^{b}$ & 0 & 0 & $1^{b}$ & 0 & 0 & 0 & 0 & 0 & 0 & 0 & $1^{b}$ \\
\hline & Low & 5 & ND & 3 & 4 & 7 & 8 & 8 & 7 & 8 & 7 & 4 & 6 & 7 & 6 \\
\hline & High & 6 & ND & 8 & 7 & 8 & 8 & 8 & 8 & 8 & 8 & 8 & 8 & 8 & 8 \\
\hline \multirow{3}{*}{ Lettuce } & Blank & $1^{b}$ & ND & 0 & 0 & $1^{b}$ & 0 & 0 & 0 & 0 & 0 & 0 & 0 & 0 & 0 \\
\hline & Low & 8 & ND & 7 & 5 & 3 & 7 & 6 & 6 & 8 & 8 & 6 & 7 & 6 & 6 \\
\hline & High & 8 & ND & 7 & 7 & 8 & 8 & 8 & 8 & 8 & 8 & 8 & 7 & 8 & 8 \\
\hline
\end{tabular}

a Inoculation of pathogenic Y. enterocolitica: Blank, negative; Low, 9 CFU / 25 ml (raw milk) or 16 CFU / 25 g (minced meat) or 110 CFU / 25 g (lettuce);

High, 59 CFU / 25 ml (raw milk) or 85 CFU / 25 g (minced meat) or 1100 CFU / 25 g (lettuce). ND, no data

${ }^{\mathrm{b}}$ False positive result 
Table 2. Results of data analysis obtained with raw milk

\begin{tabular}{|c|c|c|c|}
\hline Performance characteristic & Blank & $\begin{array}{c}\text { Low level } \\
\text { contamination }\end{array}$ & $\begin{array}{c}\text { High level } \\
\text { contamination }\end{array}$ \\
\hline & $0 \mathrm{CFU} / 25 \mathrm{~g}$ & $9 \mathrm{CFU} / 25 \mathrm{~g}$ & $59 \mathrm{CFU} / 25 \mathrm{~g}$ \\
\hline Number of participating collaborators & 14 & 14 & 14 \\
\hline Number of collaborators retained after evaluation of the data & $12^{\mathrm{a}}$ & $12^{\mathrm{a}}$ & $12^{\mathrm{a}}$ \\
\hline Number of samples & 112 & 112 & 112 \\
\hline Number of samples retained after evaluation of the data & 96 & 96 & 96 \\
\hline Sensitivity, \% & - & 68 & 96 \\
\hline Specificity, \% & 100 & - & - \\
\hline LOD $_{50}$, (95 \% confidence interval) in CFU/sample & - & $9.4(7.4$ to 12.0$)$ & \\
\hline
\end{tabular}

a Two laboratories were excluded; one due to deviation in the protocol and other due to the inconsistency in the result reporting

Table 3. Results of data analysis obtained with minced meat

\begin{tabular}{lccc}
\hline Performance characteristic & Blank & $\begin{array}{c}\text { Low level } \\
\text { contamination } \\
16 \mathrm{CFU} / 25 \mathrm{~g}\end{array}$ & $\begin{array}{c}\text { High level } \\
\text { contamination } \\
85 \mathrm{CFU} / 25 \mathrm{~g}\end{array}$ \\
\hline Number of participating collaborators & 13 & 13 & 13 \\
Number of collaborators retained after evaluation of the data & 13 & 13 & 13 \\
Number of samples & 104 & 104 & 104 \\
Number of samples retained after evaluation of the data & 104 & 77 & 104 \\
Sensitivity, $\%$ & - & - & 97 \\
Specificity, $\%$ & $96^{\text {a }}$ & 9.9 (7.8 to 12.5) & - \\
LOD $_{50},(95 \%$ confidence interval) in CFU/sample & & 104 \\
\hline
\end{tabular}

a False positives were obtained in three different laboratories (one laboratory 2 samples out of 8; two laboratories 1 sample out of 8 ) 
Table 4. Results of data analysis obtained with lettuce

\begin{tabular}{|c|c|c|c|}
\hline Performance characteristic & Blank & $\begin{array}{c}\text { Low level } \\
\text { contamination }\end{array}$ & $\begin{array}{c}\text { High level } \\
\text { contamination }\end{array}$ \\
\hline & $0 \mathrm{CFU} / 25 \mathrm{~g}$ & $110 \mathrm{CFU} / 25 \mathrm{~g}$ & $1100 \mathrm{CFU} / 25 \mathrm{~g}$ \\
\hline Number of participating collaborators & 13 & 13 & 13 \\
\hline Number of collaborators retained after evaluation of the data & 13 & 13 & 13 \\
\hline Number of samples & 104 & 104 & 104 \\
\hline Number of samples retained after evaluation of the data & $103^{a}$ & $103^{a}$ & 104 \\
\hline Sensitivity, \% & - & 81 & 98 \\
\hline Specificity, \% & $98^{b}$ & - & - \\
\hline $\mathrm{LOD}_{50}$, (95\% confidence interval) in CFU/sample & - & 63 (49 to 81$)$ & \\
\hline
\end{tabular}

${ }^{a}$ One sample at blank level and one sample at low level for one laboratory were excluded from analysis due to lack of confirmation tests.

${ }^{\mathrm{b}}$ False positives were obtained in two different laboratories (1 sample out of 8 ). 
Table 5. Total number of participants' positive results and samples analysed at each enrichment and plating step of the method

\begin{tabular}{|c|c|c|c|c|c|c|}
\hline \multirow{3}{*}{ Enrichment/plating step } & \multicolumn{6}{|c|}{ Positive results / analysed samples ${ }^{a}$ on inoculation level ${ }^{b}$} \\
\hline & \multicolumn{2}{|c|}{ Raw milk } & \multicolumn{2}{|c|}{ Minced meat } & \multicolumn{2}{|l|}{ Lettuce } \\
\hline & Low & High & Low & High & Low & High \\
\hline PSB 0 h/CIN (direct plating) & 4 / 88 (5 \%) & $7 / 88(8 \%)$ & $2 / 104$ (2 \%) & 20 / 104 (19\%) & 22 / 104 (21\%) & 67 / 104 (64 \%) \\
\hline PSB $44 \mathrm{~h} / \mathrm{CIN}$ with $\mathrm{KOH}$ & $46 / 88(52 \%)$ & $64 / 88(73 \%)$ & 79 / 104 (76 \%) & 94 / 104 (90\%) & 44 / 100 (44 \%) & 74 / 100 (74 \%) \\
\hline PSB 44 h/CIN w/o KOH & $15 / 88(17 \%)$ & $21 / 88(24 \%)$ & $3 / 104$ (3 \%) & $11 / 104$ (11\%) & $6 / 104$ (6 \%) & 3 / 104 (3 \%) \\
\hline ITC $44 \mathrm{~h} / \mathrm{CIN}$ with $\mathrm{KOH}$ & 22 / $96(23 \%)$ & 68 / 96 (71\%) & 16 / 104 (15\%) & $28 / 104$ (27\%) & 63 / 99 (64 \%) & 82 / 100 (82 \%) \\
\hline ITC 44 h/CIN w/o KOH & 12 / $88(14 \%)$ & $53 / 88(60 \%)$ & 7 / 104 (7%) & 17 / 104 (16 \%) & 26 / 104 (25\%) & $51 / 104$ (49 \%) \\
\hline \multicolumn{7}{|l|}{ Combined results: } \\
\hline PSB $44 \mathrm{~h} / \mathrm{CIN}$ with $\mathrm{KOH}$ and w/o KOH & 55 / $88(63 \%)$ & 70 / 88 (80 \%) & 79 / 104 (76 \%) & 95 / 104 (91 \%) & $44 / 100$ (44 \%) & $71 / 100$ (71 \%) \\
\hline ITC $44 \mathrm{~h} / \mathrm{CIN}$ with $\mathrm{KOH}$ and w/o KOH & 22 / 96 (23 \%) & 71 / 96 (74 \%) & 17 / 104 (16 \%) & 28 / 104 (27\%) & 62 / 103 (60\%) & 88 / 104 (85 \%) \\
\hline PSB and ITC $44 \mathrm{~h} / \mathrm{CIN}$ with $\mathrm{KOH}$ & 45 / 88 (51 \%) & 88 / 96 (82 \%) & 80 / 104 (77 \%) & 95 / 104 (91 \%) & 76 / 99 (77 \%) & 95 / 100 (95\%) \\
\hline PSB and ITC 44 h/CIN w/o KOH & $24 / 88(27 \%)$ & 58 / 88 (66 \%) & 7 / 104 (7%) & $21 / 104$ (20 \%) & 27 / 104 (26 \%) & 52 / 104 (50 \%) \\
\hline PSB $0 \mathrm{~h} / \mathrm{CIN}, \mathrm{PSB}$ and ITC $44 \mathrm{~h} / \mathrm{CIN}$ with $\mathrm{KOH}^{\mathrm{c}}$ & 52 / 96 (54 \%) & 88 / 96 (92 \%) & 80 / 104 (77 \%) & 100 / 104 (96 \%) & 83 / 103 (81 \%) & 102 / 104 (98\%) \\
\hline PSB $0 \mathrm{~h} / \mathrm{CIN}, \mathrm{PSB}$ and ITC $44 \mathrm{~h} / \mathrm{CIN}$ with $\mathrm{KOH}$ and w/o $\mathrm{KOH}^{\mathrm{d}}$ & $65 / 96(68 \%)$ & 89 / 96 (93\%) & 80 / 104 (77 \%) & $101 / 104$ (97\%) & 83 / 103 (81 \%) & $102 / 104(98 \%)$ \\
\hline \multicolumn{7}{|c|}{$\begin{array}{l}\text { a Samples from steps that were not confirmed were removed from study round with raw milk and lettuce } \\
\text { b Inoculation of pathogenic Y. enterocolitica: Blank, negative; Low, } 9 \text { CFU / } 25 \text { ml (raw milk) or } 16 \text { CFU / } 25 \mathrm{~g} \text { (minced meat) or } 110 \text { CFU / } 25 \mathrm{~g} \text { (lettuce); } \\
\text { High, } 59 \mathrm{CFU} \mathrm{/} 25 \mathrm{ml} \text { (raw milk) or } 85 \mathrm{CFU} \mathrm{/} 25 \mathrm{~g} \text { (minced meat) or } 1100 \mathrm{CFU} \mathrm{/} 25 \mathrm{~g} \text { (lettuce). }\end{array}$} \\
\hline
\end{tabular}


Table 6. Odds ratios of the treatments on the sensitivity of detection

\begin{tabular}{|c|c|c|c|c|c|}
\hline \multirow[b]{2}{*}{ Variable } & \multirow[b]{2}{*}{ Treatment } & \multicolumn{4}{|c|}{ Posterior odds ratio ${ }^{a}$ ( $95 \%$ credibility interval) } \\
\hline & & All ILS rounds & Raw milk & Minced meat & Lettuce \\
\hline$T 1$ & PSB $0 \mathrm{~h} / \mathrm{CIN}$ (direct plating) & $0.1(0.1-0.1)$ & $0.0(0.0-0.0)$ & $0.0(0.0-0.0)$ & $0.5(0.3-0.7)$ \\
\hline$T 2$ & PSB $44 \mathrm{~h} / \mathrm{CIN}$ with $\mathrm{KOH}$ & - & - & - & - \\
\hline T3 & PSB 44 h/CIN w/o KOH & $0.0(0.0-0.0)$ & $0.1(0.1-0.2)$ & $0.0(0.0-0.0)$ & $0.0(0.0-0.0)$ \\
\hline T4 & ITC $44 \mathrm{~h} / \mathrm{CIN}$ with $\mathrm{KOH}$ & $0.3(0.3-0.4)$ & $0.4(0.3-0.6)$ & $0.0(0.0-0.0)$ & $2.1(1.4-3.0)$ \\
\hline T5 & ITC 44 h/CIN w/o KOH & $0.1(0.1-0.2)$ & $0.3(0.2-0.4)$ & $0.0(0.0-0.0)$ & $0.4(0.2-0.5)$ \\
\hline T6 & PSB $44 \mathrm{~h} / \mathrm{CIN}$ with $\mathrm{KOH}$ and w/o KOH & $1.1(1.1-1.1)$ & $1.2(1.1-1.3)$ & $1.0(1.0-1.0)$ & $1.0(1.0-1.1)$ \\
\hline$T 7$ & ITC $44 \mathrm{~h} / \mathrm{CIN}$ with $\mathrm{KOH}$ and w/o KOH & $0.5(0.4-0.7)$ & $0.8(0.6-1.2)$ & $0.0(0.0-0.1)$ & $3.1(2.1-4.4)$ \\
\hline T8 & PSB and ITC $44 \mathrm{~h} / \mathrm{CIN}$ with $\mathrm{KOH}$ & $1.8(1.6-2.0)$ & $1.9(1.6-2.2)$ & $1.1(1.1-1.2)$ & $4.8(3.7-6.3)$ \\
\hline T9 & PSB and ITC $44 \mathrm{~h} / \mathrm{CIN}$ w/o KOH & $0.2(0.1-0.2)$ & $0.4(0.3-0.6)$ & $0.0(0.0-0.0)$ & $0.4(0.3-0.5)$ \\
\hline$T 10$ & PSB $0 \mathrm{~h} / \mathrm{CIN}, \mathrm{PSB}$ and ITC $44 \mathrm{~h} / \mathrm{CIN}$ with $\mathrm{KOH}$ & $2.1(1.9-2.4)$ & $1.9(1.6-2.3)$ & $1.2(1.1-1.3)$ & $7.2(5.2-9.8)$ \\
\hline$T 11$ & PSB $0 \mathrm{~h} / \mathrm{CIN}, \mathrm{PSB}$ and ITC $44 \mathrm{~h} / \mathrm{CIN}$ with $\mathrm{KOH}$ and w/o KOH & $2.8(2.3-3.3)$ & $3.1(2.4-3.9)$ & $1.3(1.1-1.4)$ & $9.8(6.8-14.0)$ \\
\hline
\end{tabular}

${ }^{a}$ Mean of 10000 iterations. Odds ratios were calculated for each treatment in relation to the reference $T 2$ 
Table 7. The effect of individual enrichment and/or plating steps and their combinations to the level of detection (LOD $\left.{ }_{50}\right)$ within method ISO 10273

\begin{tabular}{|c|c|c|c|}
\hline \multirow[b]{2}{*}{ Enrichment and/or plating step } & \multicolumn{3}{|c|}{$\mathrm{LOD}_{50}(95 \%$ confidence interval), CFU/25 $\mathrm{ml}$ or $\mathrm{g}$} \\
\hline & Raw milk & Minced meat & Lettuce \\
\hline \multicolumn{4}{|l|}{ Individual steps: } \\
\hline PSB $0 \mathrm{~h} / \mathrm{CIN}$ (direct plating) & $360(200-670)$ & $300(198-463)$ & $620(500-780)$ \\
\hline PSB 44 h/CIN with $\mathrm{KOH}$ & $21(17-26)$ & $15(12-18)$ & $380(300-480)$ \\
\hline PSB 44 h/CIN w/o KOH & $100(72-142)$ & $500(290-850)$ & $9500(4800-19000)$ \\
\hline ITC $44 \mathrm{~h} / \mathrm{CIN}$ with $\mathrm{KOH}$ & $31(25-38)$ & $140(105-195)$ & $250(200-310)$ \\
\hline ITC 44 h/CIN w/o KOH & $44(34-57)$ & $280(186-423)$ & $820(650-1100)$ \\
\hline \multicolumn{4}{|l|}{ Combination of steps: } \\
\hline PSB $0 \mathrm{~h} / \mathrm{CIN}$ and PSB $44 \mathrm{~h} / \mathrm{CIN}$ with $\mathrm{KOH}$ & $17(14-21)$ & $11(9,1-14)$ & $200(160-250)$ \\
\hline PSB 44 h/CIN with $\mathrm{KOH}$ and w/o KOH & $15(13-20)$ & $14(11-17)$ & $410(330-510)$ \\
\hline ITC 44 h/CIN with $\mathrm{KOH}$ and w/o KOH & $29(23-36)$ & $140(103-190)$ & $240(190-300)$ \\
\hline PSB $0 \mathrm{~h} / \mathrm{CIN}, \mathrm{PSB}$ and ITC $44 \mathrm{~h} / \mathrm{CIN}$ with $\mathrm{KOH}^{\mathrm{a}}$ & $12(9,6-15)$ & $11(8,4-13)$ & $68(54-89)$ \\
\hline PSB $0 \mathrm{~h} / \mathrm{CIN}, \mathrm{PSB}$ and ITC $44 \mathrm{~h} / \mathrm{CIN}$ with $\mathrm{KOH}$ and w/o KOH ${ }^{\mathrm{b}}$ & $9.4(7,4-12)$ & $9.9(7,8-13)$ & $63(49$ to 81$)$ \\
\hline
\end{tabular}

${ }^{\mathrm{a}}$ Steps represent the mandatory enrichment/plating steps of the revised EN ISO 10273 (Anonymous 20017);

${ }^{b}$ Steps include the mandatory enrichment/plating steps of the revised EN ISO 10273:2017 (Anonymous 20017) optional plating from enrichments PSB and ITC without $\mathrm{KOH}$ treatment (mandatory steps in the former version of the method, EN ISO 10273:2003, Anonymous 2003) 


\section{Supplementary tables}

Table S1: Homogeneity and stability of interlaboratory study (ILS) samples

\begin{tabular}{|c|c|c|c|c|c|c|}
\hline \multirow[b]{2}{*}{ ILS round } & \multirow[b]{2}{*}{ Level } & \multirow{2}{*}{$\begin{array}{c}\text { Inoculum, } \\
\text { CFU/25 g/ml } \\
\text { (Standard } \\
\text { deviation, CFU) }\end{array}$} & \multicolumn{4}{|c|}{ Positive samples, observed / expected ${ }^{a}$} \\
\hline & & & $\begin{array}{l}\text { Homogeneity } \\
\text { of inoculum, } \mathrm{T} 2 \\
/(\mathrm{I}-1)^{\mathrm{b}}\end{array}$ & $\begin{array}{l}\text { Homogeneity } \\
(n=20)\end{array}$ & $\begin{array}{l}\text { Stability, } \\
\text { optimal }^{\mathrm{c}}(\mathrm{n}=10)\end{array}$ & $\begin{array}{l}\text { Stability, } \\
\text { suboptimal }^{c} \\
(\mathrm{n}=10)\end{array}$ \\
\hline & Blank & 0 & & $0 / 0$ & $0 / 0$ & $0 / 0$ \\
\hline & Low & $9(3,0)$ & 0,86 & $17 / 1-20$ & $7 / 1-10$ & $6 / 1-10$ \\
\hline \multirow[t]{3}{*}{ Raw milk } & High & $59(7,7)$ & 1,33 & $20 / 20$ & $10 / 10$ & $10 / 10$ \\
\hline & Blank & 0 & & $0 / 0$ & $0 / 0$ & $0 / 0$ \\
\hline & Low & $16(4,0)$ & 1,31 & $20 / 1-20$ & $10 / 1-10$ & $10 / 1-10$ \\
\hline \multirow[t]{3}{*}{ Minced meat } & High & $85(15)$ & 1,68 & $20 / 20$ & $10 / 10$ & $10 / 10$ \\
\hline & Blank & 0 & & $0 / 0$ & $0 / 0$ & $0 / 0$ \\
\hline & Low & $110(8,6)$ & 0,52 & $20 / 1-20$ & $7^{d} / 1-10$ & $9 / 1-10$ \\
\hline Lettuce & High & $1100(11)$ & 1,60 & $20 / 20$ & $9^{d} / 10$ & $10 / 10$ \\
\hline
\end{tabular}

${ }^{\text {a }}$ Target inoculation level was at $\mathrm{LOD}_{50}$, which would result approximately 5 out of 10 samples or 10 out of 20 samples positive.

${ }^{\mathrm{b}}$ Acceptable variation between units of a batch: T2 / (I - 1) $\leq 2$ (ISO/TS 22117:2010, Annex B)

${ }^{\mathrm{c}}$ Optimal shipping conditions: Samples in transport package (containing dry ice) were kept at room temperature $\left(22\right.$ to $\left.25^{\circ} \mathrm{C}\right)$ for 20 to $24 \mathrm{~h} \mathrm{before} \mathrm{start} \mathrm{of}$ the analysis. Suboptimal shipping and storage conditions: Samples in transport package (containing dry ice) were kept at room temperature for $3 \mathrm{~d}$, the package was opened and the samples transferred for storage at $-20^{\circ} \mathrm{C}$ for 3 or $4 \mathrm{~d}$ before start of the analysis.

d) Analysis of the sample set suffered from malfunction of $\mathrm{KOH}$ reagent 
Table S2. Sensitivities on chromogenic media in relation to their parallel plating step on CIN agar

\begin{tabular}{|c|c|c|c|c|}
\hline \multirow{3}{*}{ Enrichment/plating step } & \multicolumn{4}{|c|}{ Correct positives / analysed samples ${ }^{a}$ on inoculation level } \\
\hline & \multicolumn{2}{|c|}{ Minced meat } & \multicolumn{2}{|l|}{ Lettuce } \\
\hline & Low & High & Low & High \\
\hline PSB 44 h/CIN with $\mathrm{KOH}$ & 79 / 104 (76 \%) & $94 / 104$ (90\%) & $44 / 100(44 \%)$ & $74 / 100(74 \%)$ \\
\hline PSB $44 \mathrm{~h} / \mathrm{CHROM}^{\mathrm{b}}$ with $\mathrm{KOH}$ & NA & NA & $23 / 52(44 \%)$ & $46 / 52(88 \%)$ \\
\hline PSB 44 h/YECA ${ }^{c}$ with $\mathrm{KOH}$ & NA & NA & $0 / 4$ (0\%) & $1 / 4(25 \%)$ \\
\hline PSB 44 h/CIN w/o KOH & $3 / 104$ (3 \%) & $11 / 104(11 \%)$ & $6 / 104$ (6\%) & $3 / 104$ (3 \%) \\
\hline PSB 44 h/CHROM w/o KOH & NA & NA & $1 / 32(3 \%)$ & $1 / 32(3 \%)$ \\
\hline PSB 44 h/YECA w/o KOH & NA & NA & NA & NA \\
\hline ITC $44 \mathrm{~h} / \mathrm{CIN}$ with $\mathrm{KOH}$ & $16 / 104(15 \%)$ & $28 / 104(27 \%)$ & 63 / 99 (64 \%) & 82 / $100(82 \%)$ \\
\hline ITC 44 h/CHROM with $\mathrm{KOH}$ & 11 / 56 (20 \%) & 12 / 56 (21 \%) & 2 / 8 (25\%) & 5 / 8 (63\%) \\
\hline ITC 44 h/YECA with $\mathrm{KOH}$ & $3 / 51(6 \%)$ & 7 / 48 (15\%) & NA & NA \\
\hline ITC 44 h/CIN w/o KOH & $7 / 104$ (7 \%) & 17 / 104 (16\%) & $26 / 104(25 \%)$ & $51 / 104$ (49 \%) \\
\hline ITC 44 h/CHROM w/o KOH & 4 / 56 (7 \%) & 3 / 56 (5 \%) & $0 / 8(0 \%)$ & 0 / $8(0 \%)$ \\
\hline ITC 44 h/YECA w/o KOH & $3 / 71(4 \%)$ & $11 / 68(16 \%)$ & NA & NA \\
\hline
\end{tabular}

${ }^{a}$ Samples from steps that were not confirmed were removed from analysis. NA: Not analysed.

${ }^{\mathrm{b}}$ CHROM: CHROMagar ${ }^{\mathrm{TM}}$ Y. enterocolitica (CHROMagar, Paris, France) (Renaud et al. 2013);

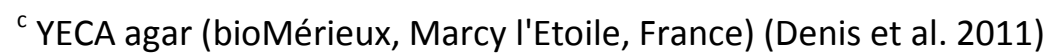


Table S3. Comparison of colony confirmation by real-time PCR method ${ }^{a}$ and biochemical confirmation pathway ${ }^{b}$

\begin{tabular}{lcccc}
\hline Performance characteristic & $\begin{array}{c}\text { No. of } \\
\text { laboratories in } \\
\text { the study }\end{array}$ & $\begin{array}{c}\text { No. of samples } \\
\text { with parallel } \\
\text { confirmation }^{c}\end{array}$ & $\begin{array}{c}\text { No. of parallel } \\
\text { confirmation } \\
\text { reactions }\end{array}$ & $\begin{array}{c}\text { No. of } \\
\text { inconsistent } \\
\text { results }\end{array}$ \\
\hline Confirmation results / raw milk & 7 & 91 & 119 & 0 \\
Confirmation results / minced meat & 8 & 122 & 130 & 0 \\
Confirmation results / lettuce & 8 & 106 & 161 & 0 \\
Confirmation results / all matrices & & 319 & 410 & 0 \\
\hline
\end{tabular}

${ }^{a}$ ISO/TS 18867:2015 (Anonymous 2015), Annex B, Methods 1 or 2 and pyrazinamidase testing

${ }^{\mathrm{b}}$ Revised ISO 10273 (Anonymous 2017)

${ }^{\mathrm{c}}$ See Figure S1 for the further explanation of alternative confirmation pathways. 


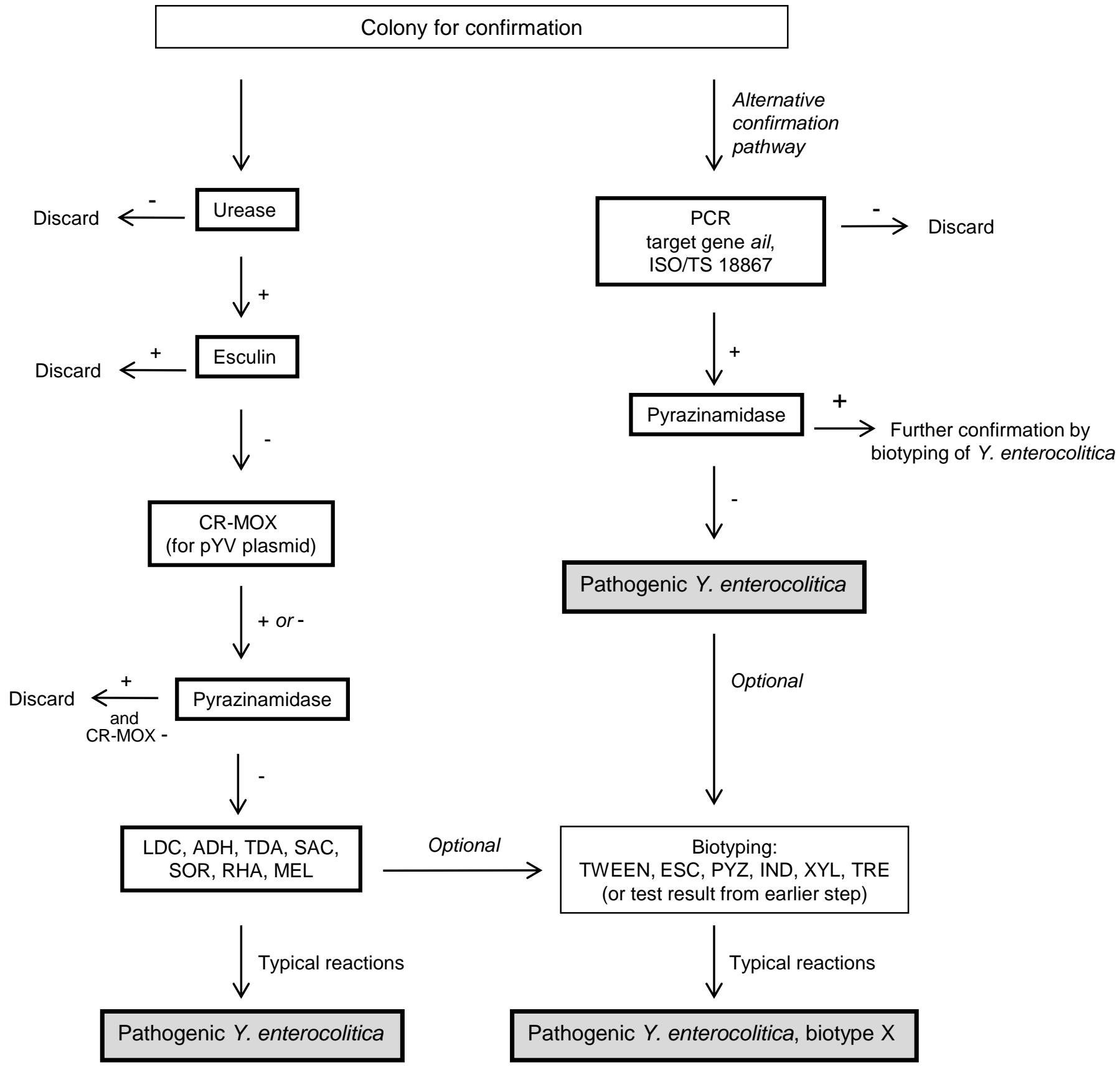


Figure S1. Diagram of the confirmation of pathogenic $Y$. enterocolitica 
Figure S2
Click here to download high resolution image
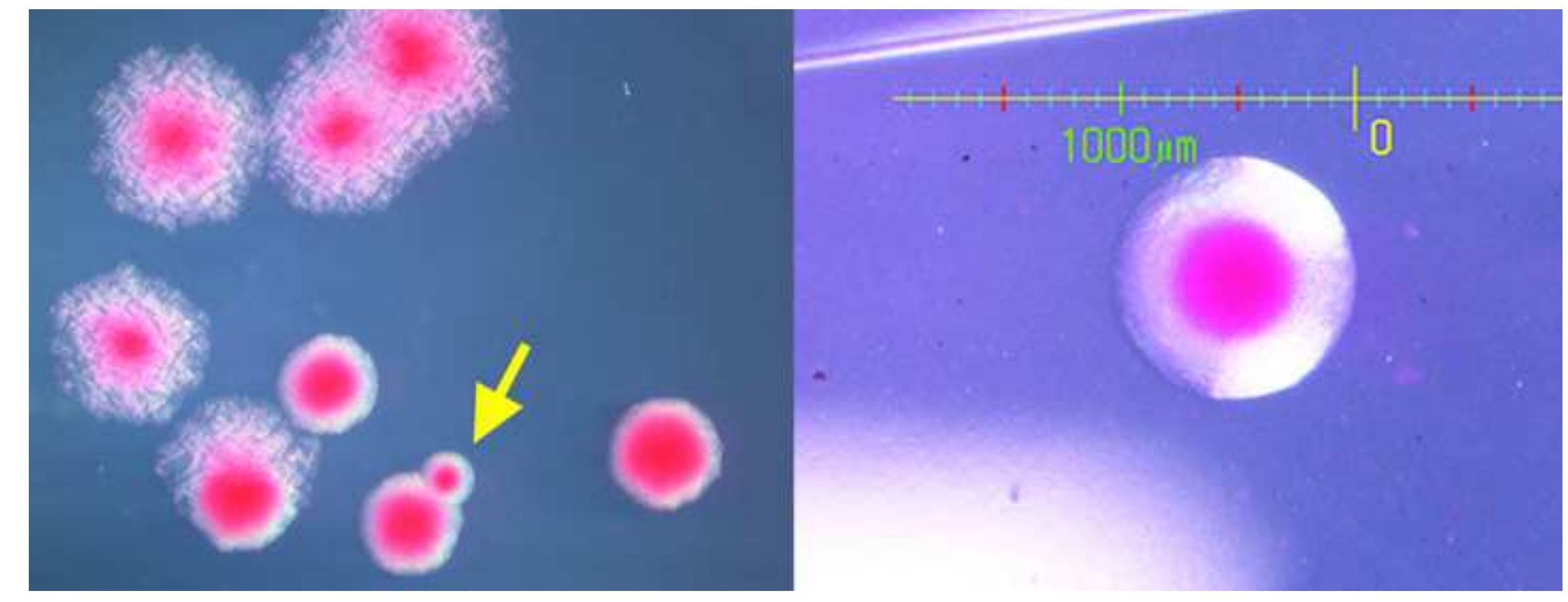

\section{Click here to download high resolution image}


Figure S2. Use of stereomicroscope in identification of pathogenic $Y$. enterocolitica colonies on $\mathrm{CIN}$ agar (incubation at $30^{\circ} \mathrm{C}$ for $24 \mathrm{~h}$ ). Left panel: one suspected colony of pathogenic $Y$. enterocolitica (indicated by arrow) among colonies of background microbiota (incl. non-pathogenic Yersinia) through stereomicroscope. On CIN agar, pathogenic Y. enterocolitica appear as small (approximately $1 \mathrm{~mm}$ ) colonies with entire, circular edge. Right panel: the characteristic morphology of the $Y$. enterocolitica bioserotype 4/O:3 colony can be identified in the magnification of the pure culture of the suspected colony on CIN. The colonies have a small, deep red sharp bordered centre ("bull's eye"). The surrounding rim is translucent or transparent and, when examined with obliquely transmitted light, non-iridescent and finely granular. 